\title{
Transcriptomic analysis of Clostridium thermocellum Populus hydrolysate-tolerant mutant strain shows increased cellular efficiency in response to Populus hydrolysate compared to the wild type strain
}

\author{
Jessica L Linville ${ }^{1,2}$, Miguel Rodriguez $\mathrm{Jr}^{2,3}$, Steven D Brown ${ }^{2,3}$, Jonathan R Mielenz ${ }^{2,3}$ and Chris D Cox ${ }^{1,2,4^{*}}$
}

\begin{abstract}
Background: The thermophilic, anaerobic bacterium, Clostridium thermocellum is a model organism for consolidated processing due to its efficient fermentation of cellulose. Constituents of dilute acid pretreatment hydrolysate are known to inhibit C. thermocellum and other microorganisms. To evaluate the biological impact of this type of hydrolysate, a transcriptomic analysis of growth in hydrolysate-containing medium was conducted on $17.5 \% \mathrm{v} / \mathrm{v}$ Populus hydrolysate-tolerant mutant (PM) and wild type (WT) strains of C. thermocellum.

Results: In two levels of Populus hydrolysate medium ( $0 \%$ and $10 \% \mathrm{v} / \mathrm{V})$, the PM showed both gene specific increases and decreases of gene expression compared to the wild-type strain. The PM had increased expression of genes in energy production and conversion, and amino acid transport and metabolism in both standard and $10 \% \mathrm{v} / \mathrm{v}$ Populus hydrolysate media. In particular, expression of the histidine metabolism increased up to 100 fold. In contrast, the PM decreased gene expression in cell division and sporulation (standard medium only), cell defense mechanisms, cell envelope, cell motility, and cellulosome in both media. The PM downregulated inorganic ion transport and metabolism in standard medium but upregulated it in the hydrolysate media when compared to the WT. The WT differentially expressed 1072 genes in response to the hydrolysate medium which included increased transcription of cell defense mechanisms, cell motility, and cellulosome, and decreased expression in cell envelope, amino acid transport and metabolism, inorganic ion transport and metabolism, and lipid metabolism, while the PM only differentially expressed 92 genes. The PM tolerates up to $17.5 \% \mathrm{v} / \mathrm{v}$ Populus hydrolysate and growth in it elicited 489 genes with differential expression, which included increased expression in energy production and conversion, cellulosome production, and inorganic ion transport and metabolism and decreased expression in transcription and cell defense mechanisms.

Conclusion: These results suggest the mechanisms of tolerance for the Populus hydrolysate-tolerant mutant strain of C. thermocellum are based on increased cellular efficiency caused apparently by downregulation of non-critical genes and increasing the expression of genes in energy production and conversion rather than tolerance to specific hydrolysate components. The wild type, conversely, responds to hydrolysate media by down-regulating growth genes and up-regulating stress response genes.
\end{abstract}

Keywords: Clostridium thermocellum, Populus hydrolysate, Inhibitor tolerance, Gene expression, Transcriptomic, RNA-seq, Consolidated bioprocessing

\footnotetext{
* Correspondence: ccox9@utk.edu

'Department of Civil and Environmental Engineering, University of

Tennessee, Knoxville, TN, USA

${ }^{2}$ Bioenergy Science Center, Oak Ridge National Laboratory, Oak Ridge, TN,

USA

Full list of author information is available at the end of the article
} 


\section{Background}

Sugars contained in plant cell walls are a potential form of renewable energy that can be transformed into liquid transportation fuels through fermentation processes. However, the sugars are present in the form of cellulosic and hemicellulosic polymers which prevents direct fermentation of biomass by common industrial microorganisms such as yeast. Cellulose is particularly insoluble and recalcitrant to biodegradation, which represents a major technological hurdle to the realization of a cellulosic biofuels industry. The presence of lignin in the plant cell wall presents additional challenges as it is not easily biodegraded, can limit access to cellulose, and has the potential to form inhibitory byproducts during biomass pretreatment. Certain thermophilic, anaerobic, Gram positive bacteria have shown the ability to biodegrade cellulose and ferment it into ethanol and other fermentation products such as acetate, lactate, formate and hydrogen, giving rise to the possibility of converting cellulose directly to transportation fuels in a single step in a process known as consolidated bioprocessing (CBP). Clostridium thermocellum is often considered to be a model organism of this class of bacteria.

Compounds generated during biomass pretreatment, hydrolysis, and microbial fermentation can have inhibitory effects on the fermenting microorganism, which decreases ethanol yields $[1,2]$ thereby rendering the process uneconomical. Improved tolerance to inhibitory compounds found in pretreated biomass hydrolysate should improve the fermentation process and increase economic feasibility of CBP. Significant clues to the mechanisms involved in adaptation to new environments, such as would be found in a $\mathrm{CPB}$ production scheme, have come from studies of gene expression in response to specific stresses [3]. The response of cells to environmental changes can provide clues to the molecular apparatuses that enable cells to adapt to new environments and the molecular mechanisms that have evolved to regulate the remodeling of gene expression that occurs in new environments [3]. By understanding the genetic basis for mechanisms of improved tolerance to inhibitors there is a possibility to rationally engineer their traits in the future [4-7].

There have been a number of studies that have analyzed the effect of various stresses associated with biofuel production, product inhibition and inhibitory compounds from pretreated biomass [8-14]. Examination of changes in the gene expression profile in response to these stresses can provide mechanistic insight to the physiological response. RNA Sequencing (RNA-seq) is an established technology for quantifying gene expression that has much greater sensitivity and dynamic range than conventional microarray technology [15]. RNA-seq is particularly relevant for controlled experiments comparing the expression in wild type and mutant strains of an organism
[16]. Moreover, combining RNA-seq with genomic data can help identify genetic loci responsible for variation in gene expression between individuals [16].

The development of a Populus hydrolysate tolerant strain of $C$. thermocellum, which grows as well in $17.5 \% \mathrm{v} / \mathrm{v}$ Populus hydrolysate as the wild type (WT) does in standard medium, has been reported [17]. Genomic analysis of the mutant strain (termed PM for Populus mutant) revealed several mutations in the strain that may be responsible for its faster growth rate and tolerance to Populus hydrolysate with selected mutations related to the transcriptional changes [17]. The extent of the growth, end product production and Populus hydrolysate tolerance was described by kinetic modeling [18]. In the present study, the WT and PM strains were grown in various concentrations of Populus hydrolysate $(0 \%$ or standard medium, $10 \%$ and $17.5 \% \mathrm{v} / \mathrm{v}$ Populus hydrolysate) and a genome-wide transcriptomic analysis was conducted at mid-log and late-log time points via RNA-seq. In addition to changes in transcription levels, post-transcriptional regulation of gene expression through the action of sRNA molecules has been demonstrated to play a key role in stress response in Clostridia [19]; however, the focus of this paper is on changes in gene regulation at the transcriptional level. Two types of comparisons were used to further elucidate the potential mechanism(s) of tolerance for the PM strain: a comparison of the strains in standard and hydrolysate media and a comparison of each strain's response to Populus hydrolysate-containing media using its gene expression profile in standard medium as a baseline.

\section{Results}

\section{Fermentative growth}

Batch fermentations were conducted for the Populus mutant (PM) and wild type (WT) strains of C. thermocellum as previously reported in Linville et al. [17]. Samples were taken at regular intervals from each fermentation unit based on their growth rate and analyzed for optical density $\left(\mathrm{OD}_{600}\right)$ and metabolite concentration by HPLC. The dry cell weight $(\mathrm{DCW})$ of the samples was determined by calibration curve (data not shown). In brief, the PM had approximately twice the growth rate when compared to the WT in standard medium $[17,18]$. The PM also produced 1.1-1.3 times more ethanol and the same amount of acetic acid than the WT under the same test conditions $[17,18]$. The dry cell weight, sugar utilization, ethanol production and acetic acid production for the fermentations are shown in (Additional file 1: Figure S1). The samples for RNA analysis were harvested from the fermentors during the midlog and late-log phase. The time points and dry cell weight of the mid-log and late-log phase can be seen in (Additional file 1: Table S1). 


\section{RNA-seq analysis}

An analysis of variance (ANOVA) was conducted on each of the independent variables separately: strain, Populus hydrolysate concentration, and time. Differentially expressed genes were defined as a 2 -fold change in expression with a false discovery rate of less than $5 \%$ $(\mathrm{p}<0.05)$. Of the 3,236 genes in $C$. thermocellum, roughly $18 \%(\mathrm{n}=574)$ showed a difference in expression between strains. Furthermore, approximately $16 \%(\mathrm{n}=$ 505 ) of the genes showed a change in expression between the three concentration comparisons. None of the genes showed a change in expression between the two time points. Since, there were no statistically significant changes in expression of individual genes between the mid-log and late-log time points, the analysis considered-between strain and between-hydrolysateconcentration comparisons to be significantly different if the expression differences were significant for either of these two time points.

Simple comparisons only consider the differences in gene expression from changing one of the three variables at a time: strain, Populus hydrolysate concentration or time. The ANOVA of the three independent variables in combination revealed approximately 55\% $(\mathrm{n}=1795)$ of the genes were differentially expressed in at least one of the simple comparisons (Additional file 2). Two types of analyses are the focus of this paper. The first analysis compares gene expression in the WT and PM strains in $0 \% \mathrm{v} / \mathrm{v}$ and $10 \% \mathrm{v} / \mathrm{v}$ Populus hydrolysate. A positive differential expression (upregulation) represents a higher expression level in the PM strain and a negative differential expression (downregulation) represents a lower expression level in the PM strain when compared to the WT strain. The second type of analysis compares gene expression under different concentrations of Populus hydrolysate within a given strain as follows: the PM in $0 \%$ versus $10 \% \mathrm{v} / \mathrm{v}$ Populus hydrolysate and $0 \%$ versus $17.5 \% \mathrm{v} / \mathrm{v}$ Populus hydrolysate, and the WT in $0 \%$ versus $10 \% \mathrm{v} / \mathrm{v}$ Populus hydrolysate. For these comparisons a positive differential expression (upregulation) represents an increase in expression level and a negative differential expression (downregulation) represents a decrease in expression level in the Populus hydrolysate compared to standard medium. Of the 1795 differentially expressed genes, 1740 are represented by these four comparisons. The remaining 55 genes are differentially expressed between the comparisons of the PM in $10 \%$ versus $17.5 \% \mathrm{v} / \mathrm{v}$ Populus hydrolysate or between the mid-log versus late-late log time points for a given condition. Genes that encode for proteins classified as hypothetical, uncharacterized or unknown function, accounting for 551 of the 1740 genes, were removed from further analysis (Additional file 3). The remaining 1189 differentially expressed genes were then assigned to one of 20 categories based on function (Additional file 4). To determine if genes within a given category were systematically regulated, the statistical significance of the odds ratio of the number of up- or down-regulated genes within a category versus the total number of up- or down- regulated genes in C. thermocellum was calculated. This process is similar to the categorical analysis of other clostridia species [12-14]. Lists of the total and differentially expressed genes by category and the total number of differentially expressed genes for each analysis are provided (Additional file 1: Table S2). Figure 1 is a pictorial representation of the five comparisons indicating the total number (including hypothetical genes) of differentially expressed genes and the categories with significant change in expression as determined by odds ratio.

Changes in gene expression level as determined by RNAseq were confirmed using real-time quantitative PCR (qPCR) for six genes from the WT versus PM in $0 \% \mathrm{v} / \mathrm{v}$ Populus hydrolysate mid-log comparison (Additional file 1: Figure S2). The coefficient of determination $R^{2}=0.92$ was obtained for comparisons of gene expression as determined by RNA-seq and qPCR (Additional file 1: Figure S2), which indicated RNA-seq data was of good quality.

\section{Discussion}

\section{Strain comparison}

The strain comparison analyzes the difference in expressed genes between the WT and PM in standard and hydrolysate media to elucidate the effect of the mutations. The 186 upregulated genes versus the 393 downregulated genes in standard medium and the 371 upregulated genes versus the 780 downregulated genes in $10 \% \mathrm{v} / \mathrm{v}$ Populus hydrolysate medium for the PM compared to the WT supports the hypothesis that the PM appears to have a more efficient cellular metabolism due to more downregulated gene expression, which leads to increased robustness regardless of the growth conditions (Figure 1). For example, PM grows at twice the rate of the WT in standard medium, indicating its greater metabolism capability or "robustness" [18]. The Populus hydrolysate tolerant phenotype of the PM is the result of two simultaneous mechanisms of action: increases in cellular repair and altered energy metabolism [17]. These mutations not only alter tolerance to the Populus hydrolysate but also alter the growth rate in standard medium suggesting a more global change in gene expression which will be evaluated by comparing the change in expression between the PM and WT strain in both standard $(0 \% \mathrm{v} / \mathrm{v}$ Populus hydrolysate) and Populus hydrolysate media.

The PM has a non-synonymous single nucleotide polymorphism (SNP) in a strongly conserved amino acid of the single copy of the rpoB gene (Cthe_2724, E885K) which encodes for a DNA directed RNA polymerase, beta subunit [17]. The beta subunit of the RNA polymerase interacts directly with both the DNA and has 
weak binding sites for the sigma factor [20]. This mutation potentially changes the specificity, activity and/or stability of the RNA polymerase which has the potential to affect a large number of genes through the promoter interaction [17,21-23]. In addition, mutations in rpo $B$ have been shown to block the uptake of aromatic compounds by the membrane transport system therefore, increasing tolerance [24]. The PM differentially expresses multiple sigma factors when compared to the WT in standard medium which can be directly linked to the overall change in expression for certain categories of genes. The differentially expressed sigma factors are listed in Table 1 and will be discussed in the context of the genes they regulate.

\section{Categories of gene with increased expression in the PM}

The PM increases the gene expression in only two categories compared to the WT in standard and Populus hydrolysate media: energy production and conversion, and amino acid transport and metabolism (Figure 1). In addition to these, the PM also increases the expression of inorganic ion metabolism and transport genes compared to the WT in $10 \% \mathrm{v} / \mathrm{v}$ Populus hydrolysate medium. The increased expression in the energy production and conversion genes may allow for the increased growth phenotype observed in the PM strain. Increases in glycolysis would lead to increases in reducing power (in the form of NADH) being available for downstream electron transport and 
Table 1 Fold change in expression of sigma factors

\begin{tabular}{|c|c|c|c|c|c|c|c|c|c|c|c|}
\hline \multirow[t]{2}{*}{ Gene name } & \multirow[t]{2}{*}{ Product } & \multicolumn{2}{|c|}{ PM vs. WT 0} & \multicolumn{2}{|c|}{ PM vs. WT 10} & \multicolumn{2}{|c|}{ PM 0 vs. 10} & \multicolumn{2}{|c|}{ PM 0 vs. 17.5} & \multicolumn{2}{|c|}{ WT 0 vs. 10} \\
\hline & & ML & LL & ML & LL & ML & LL & ML & LL & ML & LL \\
\hline the_1272 & sigma-70 region 2 domain protein & 2.34 & 1.24 & -5.64 & -3.59 & -2.20 & -1.64 & -1.38 & 1.94 & 6.00 & 2.72 \\
\hline the_0195 & Sigma-70 region 4 type 2 & 2.80 & 1.61 & -2 & 42 & -2.06 & -1.23 & 1.44 & 1.49 & 3.37 & 1.86 \\
\hline the_1438 & RNA polymerase sigma factor, sigma-70 family & 2.68 & 2.06 & 1.70 & -1.38 & -2.26 & -1.76 & -2.95 & -2.42 & -1.43 & 1.61 \\
\hline he_0890 & RNA polymerase sigma factor, sigma-70 family & 1.09 & -1.63 & -2.01 & -1.12 & 1.45 & -1.64 & -1.27 & -1.14 & -1.13 & 1.21 \\
\hline he_1809 & RNA polymerase sigma factor, sigma-70 family & 18.26 & 16.44 & 24.37 & 13.05 & -1.69 & -2.11 & -4.55 & -4.06 & -2.25 & -1.68 \\
\hline he_0446 & sigma-E processing pep & 0 & -2.21 & 1.14 & 1.26 & -1.10 & 1.45 & -1.03 & 1.51 & -1.78 & -1.92 \\
\hline the_0447 & RNA polymerase sigma-E factor & 1.90 & 2.58 & 2.15 & 1.91 & -1.56 & -1.19 & -1.30 & -2.65 & -1.77 & 1.14 \\
\hline the_0120 & RNA polymerase sigma-F factor & 1.71 & 2.01 & 2.48 & 1.96 & 1.01 & 1.15 & -1.03 & -1.22 & -1.43 & 1.18 \\
\hline the_0448 & RNA polymerase sigma-G factor & -1.79 & -2.55 & 1.09 & -1.14 & -2.10 & -1.23 & -1.56 & -1.06 & -4.11 & -2.7 \\
\hline the_1012 & RNA polymerase sigma-K factor & -3.94 & -4.74 & -2.88 & -2.96 & 1.13 & 1.20 & 1.07 & 3.57 & -1.21 & -1.3 \\
\hline the_2059 & RNA polymerase sigma-H factor & 1.45 & 1.65 & 1.86 & 1.03 & -1.30 & -1.52 & -1.41 & -2.13 & -1.66 & 1.05 \\
\hline the_0074 & RNA polymerase, sigma-24 subunit, ECF subfamily & -1.19 & -1.46 & -1.87 & -2.22 & 3.64 & 1.40 & 3.54 & 1.74 & 5.71 & 2.13 \\
\hline Cthe_0495 & RNA polymerase, sigma 28 subunit & -3.04 & -3.47 & -9.98 & -4.44 & 1.18 & 1.43 & 1.37 & 1.53 & 3.87 & 1.83 \\
\hline Cthe_2100 & transcriptional regulator, AbrB family & 2.21 & 2.48 & 8.86 & 1.29 & -2.67 & -1.16 & -5.28 & -13.66 & -10.68 & 1.66 \\
\hline Cthe_0315 & RNA polymerase sigma-I factor & -1.40 & -2.19 & -4.00 & -3.49 & -1.13 & -1.17 & -1.00 & 1.92 & 2.52 & 1.36 \\
\hline the_2975 & RNA polymerase sigma-I factor & 1.24 & 1.47 & -7.26 & -2.59 & -2.09 & -1.94 & 1.15 & 1.45 & 4.32 & 1.96 \\
\hline Cthe_0403 & RNA polymerase sigma-I factor & -1.65 & -1.92 & -5.17 & -3.64 & 1.89 & 1.76 & -1.66 & -1.08 & -1.12 & 1.83 \\
\hline
\end{tabular}

Bold values indicate significantly different expression levels as determined by ANOVA. For the PM vs. WT in 0\% and 10\% v/v Populus hydrolysate a positive/ negative value represents a higher/lower level of expression in the PM compared to the WT. For the standard medium (0\%) versus Populus hydrolysate media (10 or $17.5 \%)$ a positive/negative value represents a higher/lower expression in the hydrolysate media compared to standard medium. Values are indicated for samples collected during the mid-log (ML) and late-log (LL) growth phases.

ethanol production. The increase in ethanol production and increase in electron flux may generate sufficient $\mathrm{NAD}^{+}$to ensure increased cellular metabolism [8].

The assemblage of genes encoding proteins involved in pyruvate metabolism and end-product synthesis dictate, in part, how carbon and electrons flux is distributed between the catabolic, anabolic, and energy producing pathways of the cell [25]. C. thermocellum catabolizes glucose via the Embden-Meyerhof pathway using the "malate shunt" (Figure 2) [26-28]. Compared to the WT, the PM had a higher expression of 23 and 44 genes belonging to the energy production and conversion category in standard and Populus hydrolysate media, respectively. The PM upregulated 8 genes specific to the central metabolism and mixed-acid fermentation compared to the WT in standard medium (Figure 2 and Table 2). In $10 \% \mathrm{v} / \mathrm{v}$ Populus hydrolysate medium, the PM upregulated 10 genes along the central metabolism and mixed acid fermentation pathways compared to the WT. The PM has a mutation in the non-coding region upstream of the Cthe_0422-Cthe_0423 operon which encodes the rex (redox) repressor and the $a d h E$ alcohol dehydrogenase. This mutation may cause the observed increase in ethanol production $[17,18]$. A study of the effect of cellulose fermentation found that the central metabolism genes are typically upregulated during cellulose fermentation compared to cellobiose fermentation that the cells were grown on in this study $[12,25]$. The native upregulation of these genes by the PM may allow for the phenotypically faster growth rate.

C. thermocellum uses the hydrogenase-mediated pathway for production of molecular hydrogen to dispose the excess reducing equivalents generated during carbohydrate catabolism $[12,28]$. In the process, the Ech hydrogenase complex pump $\mathrm{H}^{+} / \mathrm{Na}^{+}$ions across the cell membrane and create proton gradients for powering ATP synthesis by ATP synthase (ATPase) [12]. The PM has a mutation in the non-coding region 127 bp upstream of the F-type ATP synthase operon (Cthe_2602 Cthe_2609) which may lead to an increase in the expression of this gene cluster in the PM compared to the WT in standard medium (Table 3) [17]. The PM also increases the expression of 4 and 8 genes in the Ech hydrogenase complex (Cthe_3013-3024) compared to the WT in standard and Populus hydrolysate media (Table 3). The effect of the increased expression of the ATPase and Ech-type hydrogenases on the electron flux in the cell is unknown at the time [17]. However, analysis of the $\mathrm{H}_{2}$ production rate of PM and WT in 0\% and 10\% v/v Populus hydrolysate media shows no significant difference [17]. In addition, regardless of the strain or growth medium, the five other hydrogen producing complexes in $C$. thermocellum are expressed at levels between 4 and 50 times greater than the Ech-type hydrogenases (data not shown) [12]. Collectively 


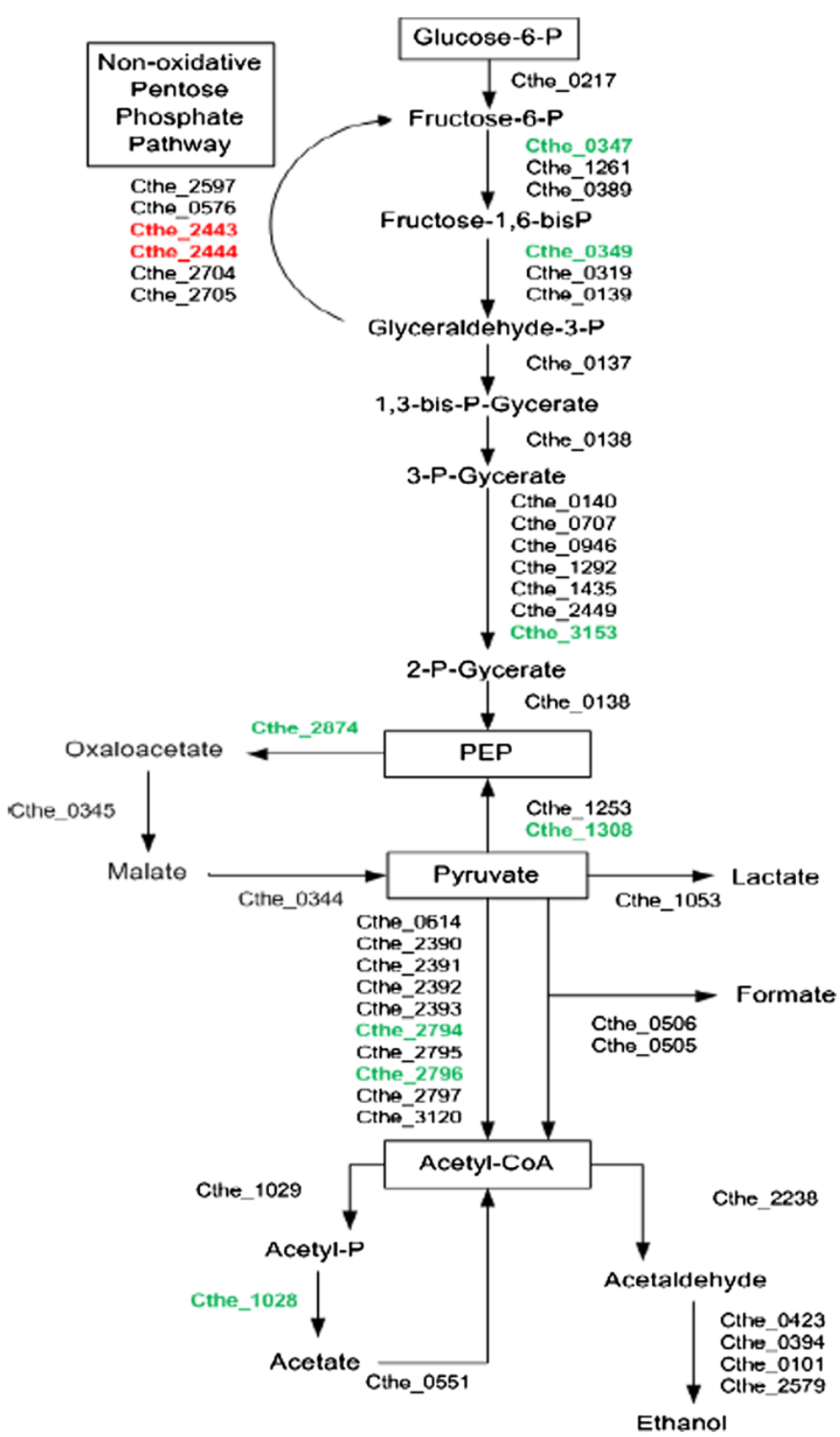

Figure 2 Central metabolism of $C$. thermocellum with differentially expressed genes between the WT and PM higlighted. Genes colored geen have greater than 2-fold higher expression and genes colored red have a greater than 2-fold lower expression in the PM than the WT in standard media. The extent of gene expression change and expression levels for the other comparisons are given in Table 2.

these results argue against the increased activity of Ech-type hydrogenase complex significantly changing the electron flux in the PM. Another possibility for this change in gene expression could be electron bifurcation which was recently found in anaerobic microbes. For example, Acetobacterium woodii employs a sodium-motive ferredoxin: $\mathrm{NAD}^{+}$-oxidoreductase (Rnf complex) that couples the exergonic electron flow from reduced ferredoxin to $\mathrm{NAD}^{+}$to establish a transmembrane electrochemical $\mathrm{Na}^{+}$gradient that then drives the synthesis of ATP via a well characterized $\mathrm{Na}^{+} \mathrm{F}_{1} \mathrm{~F}_{0}$ - ATP synthase [29]. The data showed that the complex was reduced by the [FeFe]- hydrogenase of $A$. woodii and reduction of one was strictly dependent on the presence of the other electron acceptor [29]. Clostridium kluyveri have also been shown to catalyze 
Table 2 Fold change in gene expression along the central metabolism and mixed-acid fermentation pathways

\begin{tabular}{|c|c|c|c|c|c|c|c|c|c|c|c|}
\hline \multirow[t]{2}{*}{ Gene } & \multirow[t]{2}{*}{ Product } & \multicolumn{2}{|c|}{ PM vs. WT 0} & \multicolumn{2}{|c|}{$\begin{array}{l}\text { PM vs. WT } \\
10\end{array}$} & \multicolumn{2}{|c|}{ PM 0 vs. 10} & \multicolumn{2}{|c|}{$\begin{array}{l}\text { PM } 0 \text { vs. } \\
17.5\end{array}$} & \multicolumn{2}{|c|}{ WT 0 vs. 10} \\
\hline & & $\overline{\mathrm{ML}}$ & LL & $\overline{\mathrm{ML}}$ & LL & $\overline{M L}$ & LL & $\overline{\mathrm{ML}}$ & LL & $\overline{\mathrm{ML}}$ & LL \\
\hline \multicolumn{12}{|c|}{ glucose-6-phosphate to PEP } \\
\hline Cthe_0347 & Phosphofructokinase & 1.77 & 2.59 & 2.97 & 2.13 & -1.35 & -1.31 & -1.14 & -1.49 & -2.27 & -1.07 \\
\hline Cthe_0349 & fructose-1,6-bisphosphate aldolase, class II & 1.60 & 2.49 & 3.31 & 2.50 & -1.52 & -1.41 & -1.49 & -2.03 & -3.14 & -1.42 \\
\hline Cthe_2449 & Phosphoglycerate mutase & -2.46 & -1.85 & 1.42 & -1.74 & -1.48 & -1.90 & -2.01 & -2.88 & -5.18 & -2.03 \\
\hline Cthe_3153 & alpha-ribazole phosphatase & 2.11 & 2.33 & 1.40 & 1.40 & 1.21 & 1.23 & 1.42 & -1.14 & 1.82 & 2.04 \\
\hline Cthe_0143 & Enolase & -1.23 & -1.04 & 1.63 & -1.02 & -1.11 & -1.13 & -1.05 & -2.96 & -2.22 & -1.16 \\
\hline \multicolumn{12}{|c|}{ Non-oxidative Pentose Phosphate pathway } \\
\hline Cthe_2443 & Transketolase domain-containing protein & -3.24 & -4.70 & 1.02 & -3.00 & 1.14 & -1.75 & -1.90 & -1.52 & -2.88 & -2.74 \\
\hline Cthe_2444 & Transketolase domain-containing protein & -3.47 & -4.63 & -1.15 & -3.39 & 1.26 & -1.72 & -1.63 & -1.57 & -2.41 & -2.36 \\
\hline Cthe_2705 & Transketolase central region & -1.44 & -1.33 & 2.25 & 1.19 & 1.24 & 1.21 & 1.17 & -1.81 & -2.60 & -1.32 \\
\hline \multicolumn{12}{|c|}{ PEP to Pyruvate } \\
\hline Cthe_2874 & Phosphoenolpyruvate carboxykinase [GTP] & 1.39 & 2.46 & 1.43 & 2.09 & -1.05 & -1.04 & 1.30 & 1.38 & -1.07 & 1.14 \\
\hline Cthe_0344 & malic protein NAD-binding & -1.68 & 1.06 & 1.26 & -1.10 & -1.01 & -1.14 & 1.20 & -1.27 & -2.13 & 1.02 \\
\hline Cthe_1308 & pyruvate, phosphate dikinase & 1.64 & 2.29 & -1.30 & 1.65 & -1.05 & 1.07 & 1.30 & 1.10 & 2.03 & 1.49 \\
\hline \multicolumn{12}{|c|}{ Pyruvate to Lactate/Formate/Acetyl-CoA } \\
\hline Cthe_1053 & L-lactate dehydrogenase & -1.78 & -1.25 & 1.32 & -1.02 & -1.41 & -1.27 & -1.33 & -1.16 & -3.30 & -1.55 \\
\hline Cthe_2794 & pyruvate/ketoisovalerate oxidoreductase, gamma subunit & 4.30 & 1.48 & 5.15 & 3.99 & 1.92 & 2.56 & 2.45 & 2.78 & 1.61 & -1.05 \\
\hline Cthe_2796 & $\begin{array}{l}\text { pyruvate flavodoxin/ferredoxin oxidoreductase domain } \\
\text { protein }\end{array}$ & 3.13 & 1.47 & 4.16 & 2.94 & 1.98 & 2.05 & 1.88 & 1.94 & 1.49 & 1.02 \\
\hline Cthe_0505 & formate acetyltransferase & -1.95 & -1.91 & 1.46 & -1.04 & 1.24 & 1.04 & 1.11 & -1.81 & -2.31 & -1.76 \\
\hline \multicolumn{12}{|c|}{ Acetyl-CoA to Ethanol/Acetate } \\
\hline Cthe_1028 & Acetate kinase & 1.67 & 2.57 & 3.63 & 3.05 & 2.12 & 1.26 & 2.76 & 1.50 & -1.02 & 1.06 \\
\hline Cthe_1029 & phosphate acetyltransferase & 1.54 & 1.79 & 4.01 & 3.83 & 2.42 & 1.33 & 2.73 & 1.63 & -1.08 & -1.61 \\
\hline Cthe_2238 & Aldehyde Dehydrogenase & 1.06 & 1.04 & -1.81 & -1.29 & 1.20 & 1.36 & 1.36 & 1.02 & 2.30 & 1.83 \\
\hline Cthe_0101 & iron-containing alcohol dehydrogenase & -1.35 & -1.19 & 2.19 & 1.20 & 1.22 & -1.04 & -1.18 & -1.82 & -2.43 & -1.48 \\
\hline Cthe_0423 & iron-containing alcohol dehydrogenase & 1.12 & 1.07 & 4.75 & 5.02 & 1.26 & 1.06 & 1.28 & 1.45 & -3.36 & -4.42 \\
\hline
\end{tabular}

Bold values indicate significantly different levels of expression as determined by ANOVA. For the PM vs. WT in $0 \%$ and $10 \%$ v/v Populus hydrolysate, a positive/ negative value represents a higher/lower expression level in the PM compared to the WT. For the standard medium (0\%) versus Populus hydrolysate media (10 or $17.5 \%)$ positive/negative values represents higher/lower expression levels in the hydrolysate media compared to standard medium. Values are indicated for samples collected during mid-log (ML) and late-log (LL) growth phases.

acetyl-CoA and ferredoxin-dependent formation of $\mathrm{H}_{2}$ from NADH [30].

Furthermore, sigma factor $\sigma^{\mathrm{A}}$ is the principle sigma factor present in vegetatively growing B. subtilis and other Gram-positive bacteria [31] and it directs transcription of genes important to metabolism [23]. There are 10 genes that encode for $\sigma^{\mathrm{A}}$ subunits in C. thermocellum. Three of the genes that encode for $\sigma^{\mathrm{A}}$ (Cthe_0195, Cthe_1438 and Cthe_1809) are upregulated in the PM compared to the WT in standard conditions (Table 1). The change in expression of these three sigma factors were considered significant based on the subset odds ratio of the total number of $\sigma^{\mathrm{A}}$. Oddly, the PM has a lower expression of two genes that encode for $\sigma^{\mathrm{A}}$ (Cthe_0890, and Cthe_1272) in 10\% v/v Populus hydrolysate compared to the WT; however, the PM does still increase the expression of Cthe_1809. Cthe_1809 had 18-fold greater expression level at the mid-log time point in standard media and 24-fold higher expression level at the mid-long time point in $10 \% \mathrm{v} / \mathrm{v}$ Populus hydrolysate for the PM versus WT. The higher expression level may contribute to the higher observed growth rate phenotype and energy production/conservation in the PM strain under standard conditions $[17,18]$.

Of the 163 genes that encode for various parts of the amino acid transport and metabolism, the PM upregulated a significant number of genes (20 and 37 genes) compared to the WT in standard and Populus hydrolysate media. Most significantly, the PM increased the expression of 10 of the 15 genes along the histidine metabolism pathway compared to the WT in standard medium (Table 4). Cthe_2880-Cthe_2889 is a single 
Table 3 Fold change in gene expression involved in cellular redox

\begin{tabular}{|c|c|c|c|c|c|c|c|c|c|c|}
\hline & \multicolumn{2}{|c|}{$\begin{array}{l}\text { PM vs. WT } \\
0\end{array}$} & \multicolumn{2}{|c|}{$\begin{array}{l}\text { PM vs. WT } \\
10\end{array}$} & \multicolumn{2}{|c|}{ PM 0 vs. 10} & \multicolumn{2}{|c|}{$\begin{array}{l}\text { PM O vs. } \\
17.5\end{array}$} & \multicolumn{2}{|c|}{ WT 0 vs. 10} \\
\hline & $\mathrm{ML}$ & LL & ML & LL & $\mathrm{ML}$ & LL & $\mathrm{ML}$ & LL & $\mathrm{ML}$ & LL \\
\hline \multicolumn{11}{|l|}{ Redox transcriptional repressor } \\
\hline Cthe_0422 Redox-sensing transcriptional repressor rex & 1.13 & -1.08 & 7.01 & 5.53 & 1.04 & -1.02 & -1.04 & -1.11 & -5.96 & -6.08 \\
\hline \multicolumn{11}{|l|}{ Ech-type hydrogenases } \\
\hline Cthe_3013 hydrogenase expression/formation protein HypE & 1.39 & 1.19 & 3.42 & 2.34 & -1.90 & -2.24 & 1.30 & -1.14 & 1.37 & -1.03 \\
\hline Cthe_3016 [NiFe] hydrogenase maturation protein HypF & 2.34 & 2.42 & 3.10 & 3.62 & -1.03 & -1.45 & 1.28 & 1.03 & 1.52 & 1.84 \\
\hline Cthe_3017 hydrogenase accessory protein HypB & 2.66 & 3.07 & 2.56 & 3.73 & 1.12 & -1.15 & 1.08 & 1.06 & 1.63 & 1.97 \\
\hline Cthe_3018 hydrogenase expression/synthesis HypA & 2.51 & 3.11 & 2.20 & 3.99 & 1.40 & -1.07 & 1.22 & 1.20 & 1.92 & 2.27 \\
\hline Cthe_3019 4Fe-4S ferredoxin iron-sulfur binding domain-containing protein & 2.89 & 3.12 & 1.77 & 2.98 & 1.14 & 1.03 & 1.54 & 2.02 & 1.87 & 1.08 \\
\hline Cthe_3020 NADH-ubiquinone oxidoreductase chain $49 \mathrm{kDa}$ & 2.96 & 3.83 & 1.86 & 3.15 & 1.02 & -1.03 & 1.55 & 2.07 & 1.64 & 1.18 \\
\hline Cthe_3021 ech hydrogenase, subunit EchD, putative & 4.29 & 4.79 & 2.15 & 3.03 & -1.12 & -1.09 & 1.16 & 1.71 & 1.79 & 1.46 \\
\hline Cthe_3024 NADH/Ubiquinone/plastoquinone (complex I) & 2.04 & 2.26 & 2.83 & 2.10 & -1.12 & -1.10 & -1.17 & 1.05 & -1.54 & -1.02 \\
\hline \multicolumn{11}{|l|}{ ATP synthase } \\
\hline Cthe_2602 ATP synthase subunit a & 2.77 & 3.55 & 5.58 & 3.87 & -1.21 & 1.10 & -1.35 & -1.72 & -2.44 & 1.01 \\
\hline Cthe_2603 ATP synthase subunit c & 2.27 & 2.68 & 2.66 & 4.62 & 1.11 & 1.04 & -1.04 & -1.22 & -1.06 & -1.66 \\
\hline Cthe_2604 ATP synthase subunit b & 2.48 & 2.18 & 4.03 & 4.30 & -1.01 & 1.10 & -1.23 & -1.32 & -1.64 & -1.80 \\
\hline Cthe_2605 ATP synthase F1, delta subunit & 3.55 & 2.04 & 3.86 & 2.95 & -1.06 & -1.05 & -1.77 & -2.01 & -1.15 & -1.53 \\
\hline Cthe_2606 ATP synthase F1, alpha subunit & 2.40 & 2.00 & 2.75 & 3.27 & 1.60 & 1.31 & 1.20 & 1.25 & 1.40 & -1.24 \\
\hline Cthe_2607 ATP synthase F1, gamma subunit & 2.63 & 2.09 & 2.06 & 2.95 & 1.17 & 1.20 & 1.09 & 1.49 & 1.50 & -1.18 \\
\hline Cthe_2608 ATP synthase F1, beta subunit & 2.67 & 2.65 & 3.73 & 4.36 & 1.40 & 1.37 & 1.04 & 1.05 & -1.00 & -1.20 \\
\hline Cthe_2609 ATP synthase epsilon chain & 2.94 & 2.87 & 4.11 & 4.79 & 1.12 & 1.33 & -1.21 & -1.11 & -1.24 & -1.26 \\
\hline
\end{tabular}

Bold values indicate significantly different levels of expression as determined by ANOVA. For the PM vs. WT in $0 \%$ and $10 \%$ v/v Populus hydrolysate, a positive/negative value represents a higher/lower expression level in the PM compared to the WT. For the standard medium (0\%) versus Populus hydrolysate media (10 or $17.5 \%$ ) positive/negative values represents higher/lower expression levels in the hydrolysate media compared to standard medium. Values are indicated for samples collected during mid-log (ML) and late-log (LL) growth phases.

Table 4 Fold change in gene expression in histidine metabolism pathways

\begin{tabular}{|c|c|c|c|c|c|c|c|c|c|c|c|}
\hline \multirow[t]{2}{*}{ Gene } & \multirow[t]{2}{*}{ Product } & \multicolumn{2}{|c|}{ PM vs. WT 0} & \multicolumn{2}{|c|}{ PM vs. WT 10} & \multicolumn{2}{|c|}{ PM 0 vs. 10} & \multicolumn{2}{|c|}{$\begin{array}{l}\text { PM } 0 \text { vs. } \\
17.5\end{array}$} & \multicolumn{2}{|c|}{ WT 0 vs. 10} \\
\hline & & $M L$ & LL & $M L$ & LL & $M L$ & LL & $M L$ & LL & $M L$ & LL \\
\hline Cthe_2880 & ATP phosphoribosyltransferase regulatory subunit & 98.42 & 29.12 & 98.72 & 80.51 & 1.25 & 1.01 & 1.12 & -1.06 & 1.25 & -2.73 \\
\hline Cthe_2881 & ATP phosphoribosyltransferase & 78.48 & 23.79 & 85.06 & 100.15 & 1.64 & 1.24 & 1.35 & -1.01 & 1.52 & -3.40 \\
\hline Cthe_2882 & histidinol dehydrogenase & 35.86 & 18.44 & 28.45 & 44.69 & 1.49 & 1.33 & 1.37 & 1.40 & 1.88 & -1.83 \\
\hline Cthe_2883 & histidinol-phosphate aminotransferase & 38.12 & 19.61 & 23.12 & 40.22 & 1.15 & 1.22 & 1.19 & 1.42 & 1.89 & -1.69 \\
\hline Cthe_2884 & Imidazoleglycerol-phosphate dehydratase & 7.45 & 7.71 & 17.31 & 17.09 & 1.23 & 1.25 & 1.18 & 1.27 & -1.89 & -1.77 \\
\hline Cthe_2886 & Imidazole glycerol phosphate synthase subunit hisH & 11.99 & 12.29 & 14.84 & 15.87 & 1.19 & 1.12 & 1.09 & -1.01 & -1.04 & -1.16 \\
\hline Cthe_2887 & $\begin{array}{l}\text { 1-(5-phosphoribosyl)-5-[(5-phosphoribosylamino) } \\
\text { methylideneamino] imidazole-4-carboxamide isomerase }\end{array}$ & 13.46 & 11.01 & 10.02 & 14.54 & 1.44 & 1.20 & 1.29 & 1.13 & 1.93 & -1.10 \\
\hline Cthe_2888 & Imidazole glycerol phosphate synthase subunit hisF & 12.46 & 14.23 & 10.04 & 18.19 & 1.61 & 1.30 & 1.54 & 1.24 & 1.99 & 1.02 \\
\hline Cthe_2889 & Histidine biosynthesis bifunctional protein hisIE & 12.71 & 10.80 & 6.09 & 12.49 & 1.48 & 1.29 & 1.51 & 1.28 & 3.08 & 1.11 \\
\hline Cthe_3028 & Pyridoxal-dependent decarboxylase & -11.35 & -13.46 & -7.10 & -6.92 & -2.37 & -1.04 & -3.78 & -2.89 & -3.79 & -2.02 \\
\hline Cthe_3149 & aminoacyl-histidine dipeptidase & 3.34 & 4.23 & -1.07 & 1.63 & 1.15 & 1.05 & 1.39 & 1.37 & 4.09 & 2.72 \\
\hline Cthe_1332 & Histidyl-tRNA synthetase & -1.58 & -1.89 & 1.66 & -1.18 & 1.10 & -1.03 & -1.15 & -1.62 & -2.38 & -1.64 \\
\hline
\end{tabular}

Bold values indicate significantly different levels of expression as determined by ANOVA. For the PM vs. WT in $0 \%$ and $10 \%$ v/v Populus hydrolysate, a positive/negative value represents a higher/lower expression level in the PM compared to the WT. For the standard medium (0\%) versus Populus hydrolysate media (10 or $17.5 \%$ ) positive/negative values represents higher/lower expression levels in the hydrolysate media compared to standard medium. Values are indicated for samples collected during mid-log (ML) and late-log (LL) growth phases. 
operon and is among the most highly differentially expressed genes in the PM versus WT comparison, with an average 23-fold to 31-fold increase in expression in standard and Populus hydrolysate media. The PM decreases the expression of one gene in this pathway, Cthe_3028 which converts histidine to histamine (Figure 3). De novo biosynthesis of histidine during fermentation may be constrained by the high $\mathrm{NADH} / \mathrm{NAD}^{+}$ratio during anaerobic growth and the requirement for further reduction of $\mathrm{NAD}^{+}$ in the two terminal steps of biosynthesis [17]. Histidine may be limited by the addition of furfural [17]. The PM has two mutations involved with glutamate catabolism; a possible gain in function in $\operatorname{argD}$ (Cthe_1866, E55G) and a possible loss in function in $\operatorname{proB}$ (Cthe_1766, A149T) [17]. These two mutations seem to be a beneficial shift from proline production to glutamate and arginine production in PM $[17,18,32]$. The shift in amino acid production may also assist in the increased expression in the histidine pathway since glutamate is utilized in the pathway. The PM also significantly increases the expression of 6 of the 18 genes belonging to valine, leucine and isoleucine biosynthesis,

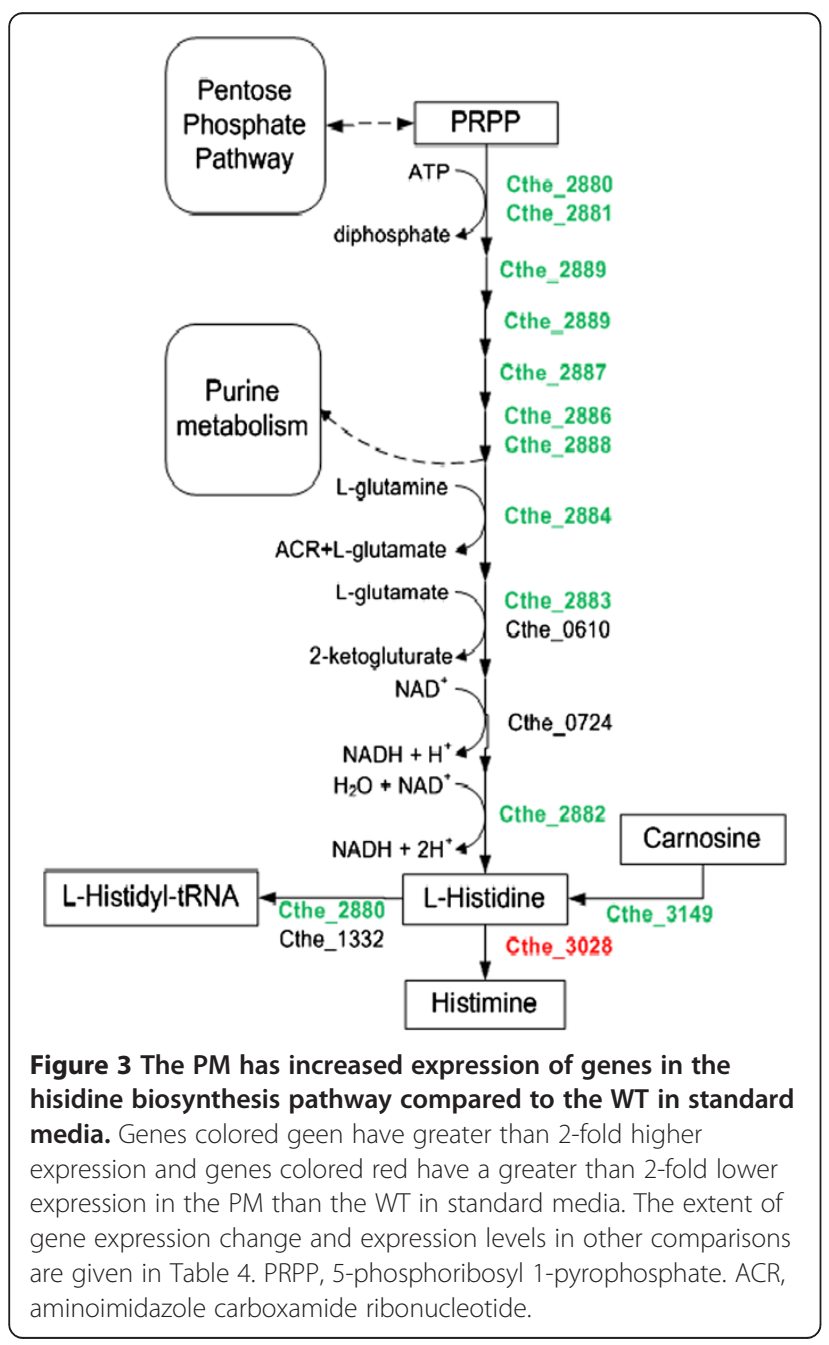

which may help balance carbon and electron flow. An increase in amino acid production can also help overcome weak acid stress $[17,18,33]$.

\section{Categories of gene with decreased expression in the PM}

There are a number of categories with decreased expression level for the PM when compared to the WT in standard medium. The downregulation of these genes may be a result of trying to conserve cellular resources and redirect them in such a way as to increase the growth rate for the PM. The downregulated categories will be discussed briefly below.

The downregulation of the cell division and sporulation genes by the PM compared to the WT in standard medium may seem counterintuitive with the faster growth rate of the PM. However, the genes in this category can be subdivided into cell division genes and sporulation genes. Independent odds ratios on the gene subsets show that only the sporulation genes were significantly downregulated by the PM in standard medium (Additional file 1: Table S3). Although the PM downregulates a greater number $(23$ compared to 20) of cell division and sporulation genes in the $10 \% \mathrm{v} / \mathrm{v}$ Populus hydrolysate medium comparison over standard medium, it is not considered significant by odds ratio due to the larger total number of genes that were down regulated in the $10 \% \mathrm{v} / \mathrm{v}$ Populus hydrolysate medium comparison. Similarly, the PM downregulates 17 genes belonging to the sporulation subcategory, however, it is not significant in the hydrolysate medium comparison as seen in Additional file 1: Table S3. There are two possible reasons that the PM downregulates the sporulation genes.

The first possible reason for the reduction in sporulation genes is a stop codon placed at the 76 amino acid in the coding region of a distantly related spoOA homologue (Cthe_3087) in the PM which should disrupt the gene function; although, the gene expression does not change significantly [17]. There is a second copy of spo0A in C. thermocellum, Cthe_0812 which is significantly downregulated by an unknown mechanism in standard conditions compared to the WT. The spoOA protein is activated when phosphorylated and has been shown to regulate sporulation in a number of clostridia [34]. Although, it is rare for C. thermocellum to go into sporulation, it has been shown that sporulation will occur under vitamin limitation, oxygen stress and switching between soluble and insoluble substrates [35]. The PM growth kinetics is consistent with other spoOA defective mutants which continue to grow under nutrient limiting conditions [36-39].

The second reason for a reduction in the expression of sporulation genes may be that the PM differentially expresses the sigma factors that control sporulation. The five known sporulation sigma factors in B. subtilis are $\sigma^{\mathrm{E}}, \sigma^{\mathrm{F}}$, $\sigma^{\mathrm{G}}, \sigma^{\mathrm{H}}$ and $\sigma^{\mathrm{K}}[31,34]$. In B. subtilis, $\sigma^{\mathrm{H}}$ is the earliest 
sporulation sigma factor [34]. $\sigma^{\mathrm{E}}$ is the mother cell-specific sigma factor and is also involved in the synthesis of $\sigma^{\mathrm{K}}$, the late-acting mother cell sigma factor [31]. Furthermore, $\sigma^{\mathrm{F}}$ dependent transcription appears to be limited to the early expression of forespore-specific genes and $\sigma^{G}$ appears to encode products that are synthesized within the forespore compartment during the later stages of sporulation to enhance spore survival and facilitate germination [31]. There are six genes that encode the various sporulation sigma factors in C. thermocellum. The PM has increased expression in $\sigma^{\mathrm{E}}$ (Cthe_0447) and $\sigma^{\mathrm{F}}$ (Cthe_0120), and decreased expression in $\sigma^{\mathrm{E}}$ (Cthe_0446) for the late-log time point, and decreased expression of $\sigma^{\mathrm{K}}$ (Cthe_1012) for both time points in the standard medium comparison (Table 1). The PM has increased expression of $\sigma^{\mathrm{E}}$ (Cthe_0447) and $\sigma^{\mathrm{F}}$ (Cthe_0120) for the mid-log time point and decreased expression of $\sigma^{\mathrm{K}}$ (Cthe_1012) for both time points in the hydrolysate medium comparison (Table 1 ). A recent study of $C$. acetobutylicum showed that $\sigma^{\mathrm{K}}$ is involved in both early and late sporulation [40]. In C. acetobutylicum sigK deletion blocks sporulation, prior to Spo0A expression and the mutant suffered from premature cell death due to excessive medium acidification in batch cultures without $\mathrm{pH}$ control [40]. The sigK defective mutant did not transition into stationary phase where cells re-assimilate the acids and produce acetone, butanol, and ethanol [40]. The results suggest a positive-feedback loop between Spo0A and $\sigma^{\mathrm{K}}$ which may be the mechanism that down regulates Cthe_0812 for the PM in standard medium compared to the WT [40].

Sporulation is an energy intensive function requiring transcription of a large number of genes. By reducing the expression of certain sporulation genes, the PM may be capable of devoting more resources to growth. Furthermore, it has been shown that $C$. thermocellum forms L-forms upon depletion of substrate [35]. It is possible the PM favors L-forms over sporulation as a mechanism to conserve energy and promote faster recovery [35]. Once the genes that control the transition to L-forms have been discovered, this hypothesis can be tested.

Microorganisms are faced with the constant threat of invading foreign DNA, by genetic elements such as phages, plasmids, transposons and genomic islands [41]. However, in controlled environments such as the laboratory conditions used during directed evolution of this strain, these defense mechanisms may play a less important role in survival. Of the genes which encode for various cell defense mechanisms, the PM downregulated the expression of 29 and 46 genes compared to the WT in standard and Populus hydrolysate media, respectively. There are three subgroups of genes that represent the majority of the cellular defense genes: CRISPR associated proteins, Hedgehog/intein hint domain proteins and phage related proteins. Together these three subgroups make up 65 of the 94 cellular defense genes (Additional file 5). Odds ratios conducted on each of the three subsets of genes indicated that the difference of expression for each sub- group was statistically significant for both standard and Populus hydrolysate media comparisons. Although, defense mechanisms have their advantages, the PM may reduce the expression of the CRISPR-associated genes and Hedgehog/ intein hint domain protein in an effort to conserve cellular resources. Since the PM did not delete the CRISPR-associated regions, it still has the ability to recognize the foreign DNA. However, the reduced expression of these two groups of genes may come at the expense of increased expression of phage associated genes. C. thermocellum has 34 genes which encode for various phage-associated proteins which are not typically considered part of the cell defense mechanisms. The PM has an average 2-fold increased expression of 6 phage associated genes compared to the WT in standard medium which was deemed significant by the odds ratio. Conversely, the PM has an average 4-fold decreased expression of 16 phage associated genes compared to the WT in Populus hydrolysate medium which was also deemed significant by the odds ratio. The change in expression may be due to the increase in the expression of phage genes in the WT standard versus Populus hydrolysate media comparison below.

C. thermocellum's rapid growth on crystalline cellulose is facilitated by a membrane bound complex, termed the cellulosome which consists of cellulases and other polysaccharide degrading enzymes assembled together in large protein complex $[12,42]$. The primary scaffoldin protein of the cellulosome complex is attached to the cell wall and binds various carbohydrate degrading enzymes [12]. Cells are tightly attached to insoluble substrates via the carbohydrate binding module (CBM) often located at the distal end of the cellulosome complex [12]. However, the composition of carbohydrate active enzymes of the cellulosome differs as a function of the growth conditions [43]. The PM has a decreased expression of 19 and 42 of the 99 genes that encode for cellulosomal components in standard and Populus hydrolysate media, respectively (Additional file 4). The statistically significant decreased expression in cellulosome genes by the PM may be an attempt to conserve energy since the cells were adapted in media containing cellobiose and soluble glucans present from the hydrolysate. It has been hypothesized that the downregulation of the cellulosome on soluble substrate such as cellobiose occurs via catabolite repression [42]. The PM has a synonymous SNP at codon 415 in RsgI6 (Cthe_2119) which is an anti- $\sigma^{\mathrm{I}}$ factor involved in regulating the expression of cellulosomal genes in the presence of xylans and cellulose [17]. It is possible that this mutation changes the specificity of the anti- $\sigma^{\mathrm{I}}$ factor and reduces the expression of the cellulosomal genes over and above the reduction that would be achieved by catabolite repression alone. 
The PM has lower expression than the WT of 31 and 54 genes that encode for cell envelope proteins in standard and Populus hydrolysate medium (Additional file 4). The PM also downregulated 21 and 50 genes that encode for cell motility in standard and Populus hydrolysate media compared to the WT. It has been proposed that the $\sigma^{\mathrm{D}}$ in $B$. subtilis controls flagellin production and possibly has a role in the expression of the methyl-accepting chemotaxis proteins [31]. Sigma factor $\sigma^{\mathrm{D}}$ (Cthe_0495) is downregulated in the PM compared to the WT in standard and Populus hydrolysate media by 3 -fold and 10-fold at the mid-log time point (Table 1) and may cause the decrease in cell motility genes. The PM also downregulated 12 genes that encode for various inorganic ion transport and metabolism proteins compared to the WT in standard medium and upregulates 17 genes in 10\% v/v Populus hydrolysate medium. However, the downregulated genes do not belong to any specific pathway. The change in expression may be due to the downregulation of inorganic ion transport and metabolism genes in the standard versus Populus hydrolysate media comparison below. The PM also downregulated 26 genes in the miscellaneous category compared to the WT in standard medium. Beyond a simple conservation of cellular resources, the benefits of reducing the expression level of genes in these categories are unclear.

\section{Hydrolysate comparison}

The Populus hydrolysate concentration comparison represents the difference in gene expression for various hydrolysate concentrations within a given strain. Inhibitory compounds from the Populus hydrolysate may affect the cell by damaging and denaturing biological molecules, resulting in adverse outcomes, including the improper folding of proteins, DNA damage, improper RNA folding and degradation, and the impairment of biophysical changes to cell membranes necessary for energy generation and the proper functioning of molecular pumps $[44,45]$. The relatively small number of differentially expressed genes (total of 92 genes) for the PM in 10\% v/v Populus hydrolysate compared to standard medium indicates that the PM strain requires relatively few changes in gene expression to adapt to the hydrolysate medium (Figure 1). This is not entirely surprising given that the PM was adapted to the hydrolysate during the directed evolution process. Even when the PM strain is placed in $17.5 \% \mathrm{v} / \mathrm{v}$ Populus hydrolysate, significant changes in expression occur in a total of 489 genes, compared to 1040 genes for the WT in 10\% v/v Populus hydrolysate (Figure 1). All of the differentially expressed genes are listed in Additional file 4. The symmetry between induced and repressed genes in the standard versus hydrolysate conditions (Figure 1) suggests that a global conservation principle, possibly imposed by finite cellular resources, is involved in the dynamics of the genetic regulatory system [46]. Analysis of the categories with a significant number of differentially expressed genes may provide insight into the differences in these two strains. In response to hydrolysate, the PM upregulates genes related to growth and downregulates genes related to adaptation or survival, whereas the WT upregulates genes related to survival and downregulates growth genes. In summary, the hydrolysate initiates a stress-link response in the WT, but not in the PM. Only one category of genes is similarly regulated between the two strains.

\section{Upregulated genes in the PM in hydrolysate media}

The genes that are significantly upregulated by the PM in hydrolysate conditions belong to energy production and conversion, amino acid transport and metabolism, inorganic ion transport and metabolism, and general transport and secretion (Figure 1).

The PM increased the expression of five energy production and conversion genes in 10\% v/v Populus hydrolysate, which represents a significant increase in expression within this category as determined by the odds ratio. The PM also increased the expression of 12 genes in this category in $17.5 \% \mathrm{v} / \mathrm{v}$ Populus hydrolysate; however, this increase was not significant due to the larger overall number of changes in gene expression. Specific differentially expressed genes related to the central metabolism can be seen in Table 3. Similarly, $C$. acetobutylicum upregulated genes related to energy production and metabolism in acetate and butyrate stress [13]. An NADPH-dependant alcohol dehydrogenase (ADH6p) was identified as one of the enzymes responsible for HMF and furfural reduction in S. cerevisiae. Furthermore, mutants with gene deletions along the pentose phosphate pathway (PPP) exhibited growth deficiency in the presence of furfural indicating that $S$. cerevisiae tolerance to furfural was associated with the activity of PPP. The increased expression in PPP genes in the PM strain in hydrolysate might assist in protecting against and repairing furfural induced damage [47].

The expression levels of the amino acid transport and metabolism genes do not change expression levels for the PM in the hydrolysate conditions. Since the PM upregulated these genes in standard medium compared to the WT, this means that the amino acid transport and metabolism genes remain elevated in the hydrolysate conditions. Conversely, C. acetobutylicum had a relatively large number of up- and down- regulated amino acid transport and metabolism related genes in acetate, butyrate and butanol stress [13]. The significantly upregulated histidine metabolism remains elevated in the hydrolysate condition with the exception of one gene Cthe_3028 which is down regulated. Histidine may be limited under 
furfural conditions so the further reduction of Cthe_3028 stops the conversion of histidine into histamine. The two terminal steps in histidine biosynthesis involve the reduction of $\mathrm{NAD}^{+}$to $\mathrm{NADH}$, a reaction that may be slowed by the high $\mathrm{NADH} / \mathrm{NAD}^{+}$ratio associated with fermentation [33]. Histidine has been shown to contribute to acid tolerance and $C$. acetobutylicum increases the expression of the histidine biosynthesis pathway when exposed to butanol and butyrate stress $[13,48]$.

The patterns of sulfur transport and metabolism of the WT in response to hydrolysate are complex. The PM upregulated 3 genes belonging to inorganic ion transport and metabolism in 10\% v/v Populus hydrolysate compared to standard medium. In $17.5 \% \mathrm{v} / \mathrm{v}$ Populus hydrolysate a total of 18 genes experienced significant changes in regulation, including both up- and down-regulation. For the PM in $17.5 \% \mathrm{v} / \mathrm{v}$ Populus hydrolysate, four of the upregulated genes belonged to the sulfate $A B C$ transporter, while 4 downregulated genes belonged to the phosphate $A B C$ transporters. This suggests an increase in sulfur metabolism within the PM cell. In addition, of the 27 genes in the cysteine and methionine metabolism pathway, 3 were upregulated in the PM in $10 \% \mathrm{v} / \mathrm{v}$ Populus hydrolysate and 6 were upregulated in $17.5 \% \mathrm{v} / \mathrm{v}$ Populus hydrolysate; both changes are significant with respect to the odds ratio (Table 5). Up regulated genes include two copies of the met $Y$ gene (Cthe_1569 and Cthe_1842) which converts serine and hydrogen sulfide into L-cysteine and Cthe_1560 and Cthe_1840 which function along the same pathway. Together, upregulation of genes related to inorganic sulfur transport and cysteine synthesis are consistent with an attempt by the cell to overcome the detrimental effects of furfural on sulfate assimilation $[13,14,33]$. However, the sulfate reduction pathway is not observed to be upregulated. It is noteworthy that both copies of the met $Y$ gene underwent mutations late in the directed evolution process that would seem to inactivate them [17]. Cthe_1569 has a stop codon inserted at amino acid 229 and Cthe_1842 has a non-synonymous SNP (P29Q) in a highly conserved region [17]. With the disruption of the cysteine synthesis pathway, cells could still obtain cysteine directly from the medium. It is possible that the mutations which resulted in the upregulation of the sulfate uptake and cysteine synthesis pathways occurred earlier in the directed evolution process and were made superfluous by the late-occurring mutations in the met $Y$ genes.

The genes that belong to the general transport category are basic $\mathrm{ABC}$ transporter and glycosyl transferase groups which are labeled with multiple COG designations. $\mathrm{ABC}$ transporters utilize ATP energy to transport inorganic ions, amino acids, hydrocarbons, polypeptides or hydrophobic compounds [44]. In some Gram-positive organisms, the ATP-binding subunit of an $\mathrm{ABC}$ system is not part of a specific transporter complex; instead, it is shared by multiple transporters [49] increasing the

Table 5 Fold change in gene expression along the cysteine and methionine metabolic pathway

\begin{tabular}{|c|c|c|c|c|c|c|c|c|c|c|c|}
\hline \multirow[t]{2}{*}{ Gene } & \multirow[t]{2}{*}{ Product } & \multicolumn{2}{|c|}{ PM vs. WT 0} & \multicolumn{2}{|c|}{$\begin{array}{l}\text { PM vs. WT } \\
10\end{array}$} & \multicolumn{2}{|c|}{ PM 0 vs. 10} & \multicolumn{2}{|c|}{$\begin{array}{l}\text { PM } 0 \text { vs. } \\
17.5\end{array}$} & \multicolumn{2}{|c|}{ WT 0 vs. 10} \\
\hline & & $\overline{\mathrm{ML}}$ & LL & $\overline{M L}$ & LL & $\overline{M L}$ & LL & $\overline{M L}$ & LL & $\overline{\mathrm{ML}}$ & LL \\
\hline Cthe_0290 & homoserine dehydrogenase & -1.03 & 1.21 & 2.33 & 1.94 & -1.78 & -1.38 & 1.35 & 1.17 & 1.45 & -1.30 \\
\hline Cthe_0580 & aminotransferase class I and II & 1.22 & 1.48 & -1.31 & 1.17 & 1.03 & -1.00 & 1.44 & 2.03 & 1.64 & 1.26 \\
\hline Cthe_0715 & S-adenosylmethionine decarboxylase proenzyme & 1.21 & 1.33 & 2.95 & -1.12 & -1.51 & -1.64 & -1.87 & -2.76 & -3.67 & -1.10 \\
\hline the_0755 & aminotransferase class | and I| & -2.42 & -1.28 & 1.59 & -1.40 & -1.75 & -1.37 & -1.60 & -2.06 & -6.77 & -1.25 \\
\hline Cthe_0961 & aspartate-semialdehyde dehydrogenase & -2.51 & -2.11 & -2.12 & -1.37 & 1.18 & 1.15 & 1.47 & 2.34 & -1.01 & -1.34 \\
\hline Cthe_1053 & L-lactate dehydrogenase & -1.78 & -1.25 & 1.32 & -1.02 & -1.41 & -1.27 & -1.33 & -1.16 & -3.30 & -1.55 \\
\hline Cthe_1200 & Adenosylhomocysteinase & -1.26 & 1.07 & 2.23 & 1.76 & 1.39 & 1.18 & 1.01 & -1.62 & -2.02 & -1.39 \\
\hline Cthe_1559 & $\begin{array}{l}\text { Cys/Met metabolism pyridoxal-phosphate-dependent } \\
\text { protein }\end{array}$ & -9.22 & -5.72 & -4.73 & -3.97 & 4.66 & 3.12 & 16.05 & 8.31 & 2.39 & 2.17 \\
\hline Cthe_1560 & Pyridoxal-5'-phosphate-dependent protein beta subunit & -6.16 & -2.97 & -3.71 & -2.65 & 6.25 & 3.41 & 15.55 & 6.43 & 3.77 & 3.05 \\
\hline Cthe_1569 & $\begin{array}{l}\text { Cys/Met metabolism pyridoxal-phosphate-dependent } \\
\text { protein }\end{array}$ & 1.02 & 1.09 & -2.06 & -1.83 & 3.94 & 2.46 & 5.21 & 4.42 & 8.24 & 4.90 \\
\hline Cthe_1728 & DNA-cytosine methyltransferase & 2.09 & 2.38 & -1.21 & 2.26 & 1.03 & -1.01 & 1.59 & 1.80 & 2.60 & 1.04 \\
\hline Cthe_1749 & DNA-cytosine methyltransferase & 1.08 & -1.12 & -5.98 & -2.41 & -1.08 & 1.20 & 1.13 & 1.46 & 5.95 & 2.58 \\
\hline Cthe_1840 & cysteine synthase A & -1.52 & -1.21 & 3.14 & 2.17 & 1.37 & 1.27 & 1.83 & -1.27 & -3.48 & -2.07 \\
\hline Cthe_1842 & O-acetylhomoserine/O-acetylserine sulfhydrylase & -1.68 & -1.54 & -1.10 & 1.52 & 1.51 & 1.16 & 2.46 & 1.75 & -1.02 & -2.01 \\
\hline
\end{tabular}

Bold values indicate significantly different levels of express as determined by ANOVA. For the PM vs. WT in $0 \%$ and $10 \% \mathrm{v} / \mathrm{v}$ Populus hydrolysate, a positive/negative value represents a higher/lower expression level in the PM compared to the WT. For the standard medium (0\%) versus Populus hydrolysate media (10 or $17.5 \%)$ positive/negative values represents higher/lower expression levels in the hydrolysate media compared to standard medium. Values are indicated for samples collected during mid-log (ML) and late-log (LL) growth phases. 
efficiency of the cell. The PM in $17.5 \%$ v/v Populus hydrolysate upregulated 16 of the 143 genes that encode for various transport genes compared to standard medium. This may allow for faster transport of compounds into the cell or inhibitors out of the cell, allowing the faster growth phenotype (Additional file 4).

\section{Downregulated genes in the PM in hydrolysate media}

A change in the environment causes a response of the genetic network which in turn allows efficient plastic adaptation of cellular metabolism to a broad range of unforeseen challenges [46]. Increased transcriptional flexibility allows the cells to address challenges on physiological timescales (not through new mutations) [46]. The PM in 10\% v/v Populus hydrolysate decreases the expression of 8 transcription genes, and in $17.5 \% \mathrm{v} / \mathrm{v}$ Populus hydrolysate it decreases the expression of 22 genes (Additional file 4). In addition the PM in 10\% v/v Populus hydrolysate decreases the expression of four genes in the cell defense mechanism category which was determined significant by the odds ratio because of the small total number of genes being differentially expressed. Cell defense mechanisms and the ability to rapidly change its transcriptional profile in response to changing environments normally contribute to cell fitness; however, these traits may be less advantageous in a steadily-maintained, pure-culture laboratory environment. As a result, the PM may be decreasing expression of cell defense and transcriptional genes as an energy saving mechanism.

\section{Upregulated genes in the WT in hydrolysate medium}

The WT in hydrolysate medium significantly upregulates two categories of genes that relates to survival mechanisms: cell defense mechanisms and cell motility genes. The WT already had a higher expression of the cell defense mechanism genes compared to the PM in standard medium which is further increased in hydrolysate medium. In $10 \% \mathrm{v} / \mathrm{v}$ Populus hydrolysate the WT increased the expression of 38 cellular defense genes compared to standard conditions (Additional file 4). The WT has an average 2-fold higher expression of 8 genes that encode Hedgehog/intein hint domain proteins and 18 phage-associated proteins in hydrolysate medium compared to standard medium. These increases are possibly part of a programmed cell response to the general deterioration of the cell health in hydrolysate conditions. While these increases in gene expression environment may help the cell to survive in a natural environment, they drain resources away from central metabolism and ethanol production. The WT in $10 \% \mathrm{v} / \mathrm{v}$ Populus hydrolysate also increases the expression of 44 cell motility genes and upregulates the expression of sigma factor $\sigma^{\mathrm{D}}$ by 3 -fold (Table 1). The increase in motility of the
WT in response to hydrolysate may be an attempt by the cell to swim away from unfavorable environments (Additional file 4). In contrast, the PM may not see the hydrolysate conditions as an unfavorable environment and further conserves energy by reducing the expression of the cell motility genes. However, a transcriptional analysis of Clostridium beijerinckii found that the genes were downregulated during the switch from acidogenesis to solventogenesis during fermentation [11]. Furthermore, C. acetobutylicum also downregulates cell motility genes in acetate stress but increases the expression in butyrate stress [13].

\section{Downregulated genes in the WT in hydrolysate}

The WT in $10 \% \mathrm{v} / \mathrm{v}$ Populus hydrolysate medium downregulates the expression of the sigma factor $\sigma^{\mathrm{A}}$ gene Cthe_1809 by 2 -fold compared to standard medium, which may contribute to the observed slower growth phenotype. Since the change in expression of Cthe_1809 is closely related to the observed growth rates in both the WT and PM, it may be one of the more important genes that encode for sigma factor $\sigma^{\mathrm{A}}$ in C. thermocellum. The WT in $10 \% \mathrm{v} / \mathrm{v}$ Populus hydrolysate does upregulate a sigma 70 region 2 domain protein; however, the protein is approximately half the length of the genes encoding for the RNA polymerase sigma factors; therefore, its exact function is unknown. Although, the WT in $10 \% \mathrm{v} / \mathrm{v}$ Populus hydrolysate does not decrease the overall expression of the energy production and conversion genes compared to standard medium, it does significantly down regulate the operon Cthe_0422-3. The wild type strain of $C$. thermocellum has shown a similar response where genes Cthe_0422-0432 were the most strongly downregulated upon exposure to furfural [14]. C. acetobutylicum also downregulates rex, a regulator of solventogenesis, under butyrate stress [48].

The WT in $10 \% \mathrm{v} / \mathrm{v}$ Populus hydrolysate decreases the expression of 37 genes in the cell envelope category compared to standard medium (Additional file 4). The WT also downregulated 11 of the 45 genes belonging to lipid degradation and biosynthesis in this comparison (Additional file 4). Organic solvents can damage the membrane structure and destabilize the function of its associated proteins [50]. Lipoprotiens are proposed to maintain the structure and function of bacterial cell envelopes [51]. C. acetobutylicum is inhibited by solvents which change the lipid composition and disrupts the cell membrane fluidity [50,51]. Transcriptomic analysis of $C$. acetobutylicum found that genes with cell envelope associated functions were the largest group to be up- and down- regulated in butanol stress conditions; however, genes involved with lipid biosynthesis were upregulated $[50,51]$. The reduction of cell envelope and lipid degradation and biosynthesis pathways suggests that the WT 
does not have the energy required to exert the elaborate and highly sophisticated regulation of these pathways in $10 \% \mathrm{v} / \mathrm{v}$ Populus hydrolysate[52].

The WT also downregulated a significant number of amino acid transport and metabolism genes (33 genes) in $10 \% \mathrm{v} / \mathrm{v}$ Populus hydrolysate compared to the standard medium (Additional file 4). However, the change in gene expression did not belong to a specific pathway. The WT downregulates 19 genes belonging to inorganic ion transport and metabolism in 10\% v/v Populus hydrolysate compared to standard medium including all 8 $A B C$ transporter genes that were increased in the PM hydrolysate comparisons (Additional file 4). This possibly reduces the amount of sulfate-derived sulfur and phosphate available in the cell. However, the fact that the WT could obtain cysteine directly from the media may have reduced its need to transport sulfate for synthesis of sulfur-containing amino acids, allowing more of the NADPH to be allocated to furfural oxidation [33].

\section{Similarly expressed category}

The PM in $17.5 \% \mathrm{v} / \mathrm{v}$ Populus hydrolysate increases the expression level of 14 genes encoding for the cellulosome. Similarly, the WT in 10\% v/v Populus hydrolysate increases the expression level of 30 genes encoding for the cellulosome. The majority of the genes with increased expression belong to various glycoside hydrolase (GH) families. The various $\mathrm{GH}$ families encode for endo- and exoglucanases used to degrade the cellulose components $[12,42]$. The PM in $17.5 \%$ v/v Populus hydrolysate increases the expression of $8 \mathrm{GH}$ family proteins, and the WT in 10\% v/v Populus hydrolysate increases the expression of $18 \mathrm{GH}$ family proteins. Populus hydrolysate does not contain any solid cellulose or hemi-cellulose; however, it does contain significant amounts of other soluble sugars from the original pretreated biomass. The concentration of sugars in the full (100\%) Populus hydrolysate include glucose $(22.7 \mathrm{~g} / \mathrm{L})$, xylose $(42.7 \mathrm{~g} / \mathrm{L})$, arabinose $(1.84 \mathrm{~g} / \mathrm{L})$, and mannose $(6.34 \mathrm{~g} / \mathrm{L})$ [17]. These molecules may play the role of signaling molecules in the regulation of cellulosomal gene activity, thereby accounting for the greater expression of cellulosomal genes in hydrolysate media [53].

\section{Conclusion}

A summary of the major mutations and related changes in gene expression or pathway activity and associated phenotypes that impart hydrolysate tolerance is shown in a conceptual model of the PM strain in Figure 4. No single mutation could explain the performance difference of the two strains; rather, several mutations each seem to impart small advantages that cumulatively contribute to the tolerance phenotype of the PM. Mutations contributed to diverted carbon and electron flows, interruption of the sporulation mechanism, modifications to the transcriptional machinery potentially leading to widespread changes in gene expression, and efficiencies related to decreases in cellulosome and cysteine synthesis as a result of the cell adapting to the laboratory growth conditions.

The greater number of genes with decreased expression versus increased expression for the PM compared to the WT in standard and Populus hydrolysate media suggests increased cellular efficiency in the PM strain of C. thermocellum. The PM increases expression in the energy production and conversion category and in the histidine biosynthesis pathway compared to the WT in standard medium. The PM also increased the expression of genes belonging to the inorganic ion transport and metabolism category compared to the WT in $10 \% \mathrm{v} / \mathrm{v}$ Populus hydrolysate. The PM has a decreased expression in a number of functional gene categories (sporulation (standard medium only), cell defense mechanisms, cell envelope biogenesis, cell motility, cellulosome, inorganic ion transport and metabolism (standard medium only) and miscellaneous genes (standard medium only)) allowing for greater efficiency. The high similarity in gene expression of the PM compared to the WT in both standard and Populus hydrolysate media may be due to the few changes in gene expression of the PM in the standard versus Populus hydrolysate media comparison. The PM strain grown in hydrolysate media versus standard medium showed fewer differentially expressed genes than the WT strain when grown in the same two conditions suggesting that there is a more targeted response to the Populus hydrolysate by the PM strain than the WT strain. The PM upregulates genes related to growth processes and downregulates genes related to survival mechanism in the hydrolysate conditions. The WT had the opposite response when placed in the hydrolysate medium. These expression level changes for the PM may be detrimental to survival in natural environments but allowed for the better growth in the laboratory environment in which the strain was evolved, thus likely allowing for better survival and bioconversion efficiency in future production facilities producing biofuels.

\section{Methods}

\section{Strain and culture conditions}

C. thermocellum ATCC 27405 was obtained from Prof. Herb Strobel, University of Kentucky collection and denoted as the wild type (WT) strain. A Populus hydrolysate-tolerant strain, referred to as the Populus Mutant (PM) strain was developed from the WT strain and has been previously described [17]. Media, Populus hydrolysate, and culture conditions, fermentation procedures, RNA extraction and isolation techniques, sequencing procedures, and RNA expression analysis were 


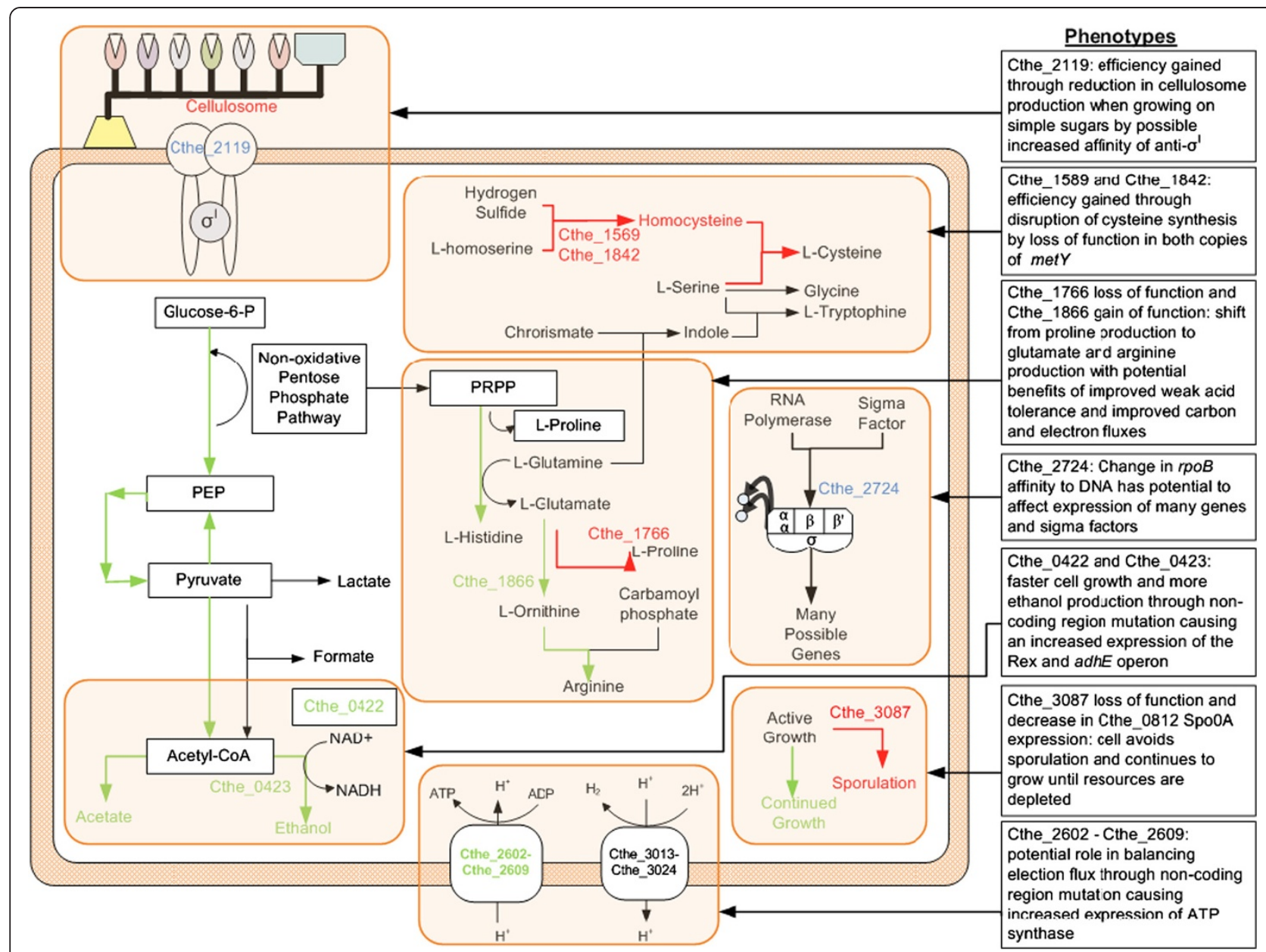

Figure 4 Summary of mutations and resulting changes in gene expression and phenotypes in the PM. Pathways (and related mutations in specific genes) with increased (green) or decreased (red) expression or functionality are shown. Mutations shown in blue do not lead to a change in gene expression but affect the affinity of the protein. The resulting phenotypic changes leading to hydrolysate tolerance are also shown.

previously described [17]. The sequenced reads NCBI study accession number is SRP024324.

\section{RNA analysis}

JMP Genomics Version 10 (SAS, Cary, NC) was used to analyze the gene expression data. Raw count data was log-2 transformed and normalized by the Upper Quartile Scaling method [54,55]. Two samples were removed from subsequent analysis due to poor data quality. An analysis of variance (ANOVA) test was conducted on each independent variable and the three independent variables together in simple comparisons using a false discovery rate method of nominal $\alpha, \mathrm{p}<0.05$. For the simple comparisons, genes were considered significantly differently expressed at a $\log -2$ difference greater than 1 (representing a 2-fold change in expression) and a $-\log 10(\mathrm{p})$ was greater than 2.126. Further analysis was conducted based on an expanded version of Clusters-of-Orthologous groups (COGs) $[12,56]$. The new annotation of $C$. thermocellum lists the
JGI categorizations which do not correspond directly to COG categories. ORNL computational biology group has also defined COG categories for 1928 genes in the new annotation of $C$. thermocellum. Both can be found here: http://genome.ornl.gov/microbial/cthe/ [55]. Additional categories were assigned for subcategories of COGs such as cellulosomal genes and transport and secretion genes. Genes were initially assigned to COGs during the annotation using RPS Blast and refined via manual curation as shown in (Additional file 1: Table S2). The full list of genes with category definition can be found in Additional file 5. To determine the significance of up or down regulation within a given category, an odds ratio of the number of up- or down-regulated genes in a category versus the total number of up- or down- regulated genes across the genome was used with a normally distributed $95 \%$ confidence interval $(\alpha=0.05)$. Odds ratios of certain additional subsets of genes were conducted to further determine significance [57]. 


\section{Quantitative-PCR (qPCR) analysis}

RNA-seq data were validated using real-time qPCR, as described previously $[7,8]$, except that the Bio-Rad MyiQ2 Two-Color Real-Time PCR Detection System (Bio-Red Laboratories, CA) and Roche FastStart SYBR Green Master (Roche Applied Science, IN) were used for this experiment. Six genes were analyzed using qPCR from cDNA derived from the mid-log time point samples for the WT and PM in standard media.

\section{Additional files}

Additional file 1: Supplemental Information. Contains all supplementary tables and figures.

Additional file 2: All statistically significant differentially expressed genes. Contains an Excel file with all of the results of the ANOVA test for all 1,795 statistically significant differentially expressed genes based on all possible simple comparisons.

Additional file 3: Significantly differentially expressed hypothetical proteins. Contains an Excel file with the 551 genes that encode hypothetical proteins, pseudo genes, and genes of unknown function.

Additional file 4: Significantly differentially expressed genes with category designation. Contains an Excel file with the 1189 genes that were significantly differentially expressed along with the category designation assigned by this analysis.

Additional file 5: Genes and category definitions. Contains an Exce file with one tab describing how the 20 categories define in this manuscript relate to JGI color categories and COGs. The other tab lists the 2,312 genes with known function that was placed into one of the 20 categories.

\section{Competing interests}

CDC has a financial interest (stock ownership) in Renmatix, Inc. Renmatix is developing technology to produce sugars from biomass via abiotic processes. He acquired stock by exercising options awarded to him as compensation for providing technical advice in the early history of the company. He no longer has any relationship with the company other than stock ownership. It is unlikely that he would be able to affect the future value of the stock through this publication, even if he were motivated to do so. CDC is the Director of the Institute for a Secure and Sustainable Environment which provided funding to support JLL through institutional funds that he has been entrusted to administer. This does not alter our adherence to all the BMC Microbiology policies on sharing data and materials.

\section{Authors' contributions}

$J L L$ conceived of the study, participated in the design of experiments, performed all experiments, analyzed and interpreted data. MR conceived of the study, participated in the design of experiments and contributed to the fermentation experiments. SDB conceived of the study, participated in the design of experiments, contributed to the analysis and interpretation of the data. JRM conceived of the study, participated in the design of experiments, contributed to the analysis and interpretation of the data. CDC conceived of the study, participated in the design of experiments, contributed to the analysis and interpretation of the data. All authors read and approved the final manuscript.

\section{Acknowledgements}

The authors thank Dawn M. Klingeman and Courtney M. Johnson for assistance with RNA purification; Dawn M. Klingeman and Charlotte M. Wilson for qPCR and PCR preparation and analysis and Qiang He and Chris Hemme for assistance with transcriptome analysis. RNA-Seq data was generated by the U.S. Department of Energy (DOE) Joint Genome Institute, which is supported by the Office of Science of the under contract no. DE-AC0205CH11231. This research was supported by the BioEnergy Science Center, a Department of Energy Bioenergy Research Center supported by the Office of Biological and Environmental Research in the Department of Energy Office of Science. Additional support was provided by the Institute for a Secure and Sustainable Environment at the University of Tennessee. Oak Ridge National Laboratory is managed by UT-Battelle, LLC, for the DOE under Contract DE-AC05-00OR22725. The funders had no role in study design, data collection and analysis, decision to publish, or preparation of the manuscript.

\section{Author details}

${ }^{1}$ Department of Civil and Environmental Engineering, University of Tennessee, Knoxville, TN, USA. ${ }^{2}$ Bioenergy Science Center, Oak Ridge National Laboratory, Oak Ridge, TN, USA. ${ }^{3}$ Biosciences Division, Oak Ridge National Laboratory, Oak Ridge, TN, USA. ${ }^{4}$ Center for Environmental Biotechnology, University of Tennessee, Knoxville, TN, USA.

Received: 14 October 2013 Accepted: 8 August 2014 Published: 16 August 2014

\section{References}

1. Palmqvist $E$, Hahn-Hagerdal B: Fermentation of lignocellulosic hydrolysates: I: inhibition and detoxification. Bioresour Technol 2000, 74(1):17-24.

2. Palmqvist $E$, Hahn-Hagerdal B: Fermentation of lignocellulosic hydrolysates: II: inhibitors and mechanisms of inhibition. Bioresour Technol 2000, 74(1):25-33.

3. Causton HC, Ren B, Koh SS, Harbison CT, Kanin E, Jennings EG, Lee TI, True $H \mathrm{~L}$, Lander ES, Young RA: Remodeling of yeast genome expression in response to environmental changes. Mol Biol Cell 2001, 12(2):323-337.

4. Hirasawa T, Furusawa C, Shimizu H: Saccharomyces cerevisiae and DNA microarray analyses: what did we learn from it for a better understanding and exploitation of yeast biotechnology? App/ Microbiol Biotechnol 2010, 87(2):391-400.

5. Bergemann TL, Wilson J: Proportion statistics to detect differentially expressed genes: a comparison with log-ratio statistics. BMC Bioinformatics 2011, 12:228.

6. Brown SD, Guss AM, Karpinets TV, Parks JM, Smolin N, Yang SH, Land ML, Klingeman DM, Bhandiwad A, Rodriguez M, Ranab B, Shao XJ, Mielenz JR, Smith JC, Keller M, Lynd LR: Mutant alcohol dehydrogenase leads to improved ethanol tolerance in Clostridium thermocellum. Proc Natl Acad Sci U S A 2011, 108(33):13752-13757.

7. Yang SH, Land ML, Klingeman DM, Pelletier DA, Lu TYS, Martin SL, Guo HB, Smith JC, Brown SD: Paradigm for industrial strain improvement identifies sodium acetate tolerance loci in Zymomonas mobilis and Saccharomyces cerevisiae. Proc Natl Acad Sci U S A 2010, 107(23):10395-10400.

8. Yang SH, Giannone RJ, Dice L, Yang ZMK, Engle NL, Tschaplinski TJ, Hettich RL, Brown SD: Clostridium thermocellum ATCC27405 transcriptomic, metabolomic and proteomic profiles after ethanol stress. BMC Genomics 2012, 13:336.

9. $\quad$ Peng YF, Luo YM, Yu TT, Xu XP, Fan KQ, Zhao YB, Yang KQ: A Blue Native-PAGE analysis of membrane protein complexes in Clostridium thermocellum. BMC Microbiol 2011, 11(1):22.

10. He MX, Wu B, Shui ZX, Hu QC, Wang WG, Tan FR, Tang XY, Zhu QL, Pan K, Li Q, Su XH: Transcriptome profiling of Zymomonas mobilis under furfural stress. Appl Microbiol Biotechnol 2012, 95(1):189-199.

11. Wang Y, Li XZ, Mao YJ, Blaschek HP: Genome-wide dynamic transcriptional profiling in Clostridium beijerinckii NCIMB 8052 using single-nucleotide resolution RNA-Seq. BMC Genomics 2012, 13:102.

12. Raman B, McKeown CK, Rodriguez M, Brown SD, Mielenz JR: Transcriptomic analysis of Clostridium thermocellum ATCC 27405 cellulose fermentation. BMC Microbiol 2011, 11:134

13. Alsaker KV, Paredes C, Papoutsakis ET: Metabolite stress and tolerance in the production of biofuels and chemicals: gene-expression-based systems analysis of butanol, butyrate, and acetate stresses in the anaerobe Clostridium acetobutylicum. Biotechnol Bioeng 2010, 105(6):1131-1147.

14. Wilson CM, Yang SH, Rodriguez M, Ma Q, Johnson CM, Dice L, Xu Y, Brown SD: Clostridium thermocellum transcriptomic profiles after exposure to furfural or heat stress. Biotechnol Biofuels 2013, 6(1):131.

15. Marioni JC, Mason CE, Mane SM, Stephens M, Gilad Y: RNA-seq: an assessment of technical reproducibility and comparison with gene expression arrays. Genome Res 2008, 18(9):1509-1517.

16. Oshlack A, Robinson MD, Young MD: From RNA-seq reads to differential expression results. Genome Biol 2010, 11(12):10.

17. Linville JL, Rodriguez M, Land M, Syed MH, Engle NL, Tschaplinski TJ, Mielenz JR, Cox CD: Industrial robustness: understanding the mechanism 
of tolerance for the Populus hydrolysate-tolerant mutant strain of Clostridium thermocellum. Plos One 2013, 8(10):16.

18. Linville $\mathrm{J}$, Rodriguez M, Mielenz JR, Cox CD: Kinetic modeling of batch fermentation for Populus hydrolysate-tolerant mutant and wild type strains of Clostridium thermocellum. Bioresour Technol 2013, 147:605-613.

19. Venkataramanan KP, Jones SW, McCormick KP, Kunjeti SG, Ralston MT, Meyers BC, Papoutsakis ET: The Clostridium small RNome that responds to stress: the paradigm and importance of toxic metabolite stress in C. acetobutylicum. BMC Genomics 2013, 14:16.

20. Burgess RR, Anthony L: How sigma docks to RNA polymerase and what sigma does. Curr Opin Microbiol 2001, 4(2):126-131.

21. Moeller R, Vlasic I, Reitz G, Nicholson WL: Role of altered rpoB alleles in Bacillus subtilis sporulation and spore resistance to heat, hydrogen peroxide, formaldehyde, and glutaraldehyde. Arch Microbiol 2012, 194(9):759-767.

22. Alper $\mathrm{H}$, Stephanopoulos $\mathrm{G}$ : Global transcription machinery engineering: a new approach for improving cellular phenotype. Metab Eng 2007, 9(3):258-267.

23. Boor KJ: Bacterial stress responses: what doesn't kill them can make them stronger. PLoS Biol 2006, 4(1):e23.

24. Hosokawa K, Park NH, Inaoka T, Itoh Y, Ochi K: Streptomycin-resistant (rpsL) or rifampicin-resistant (rpoB) mutation in Pseudomonas putida KH146-2 confers enhanced tolerance to organic chemicals. Environ Microbiol 2002, 4(11):703-712.

25. Carere CR, Rydzak T, Verbeke TJ, Cicek N, Levin DB, Sparling R: Linking genome content to biofuel production yields: a meta-analysis of major catabolic pathways among select $\mathrm{H}-2$ and ethanol-producing bacteria. BMC Microbiol 2012, 12:295

26. Lamed R, Zeikus JG: Ethanol production by thermophilic bacteria: relationship between fermentation product yields of and catabolic enzyme activities in Clostridium thermocellum and Thermoanaerobium brockii. J Bacteriol 1980, 144(2):569-578.

27. Deng Y, Olson DG, Zhou JL, Herring CD, Shaw AJ, Lynd LR: Redirecting carbon flux through exogenous pyruvate kinase to achieve high ethanol yields in Clostridium thermocellum. Metab Eng 2013, 15:151-158.

28. Rydzak T, Levin DB, Cicek N, Sparling R: End-product induced metabolic shifts in Clostridium thermocellum ATCC 27405. Appl Microbiol Biotechnol 2011, 92(1):199-209.

29. Schuchmann $K$, Muller $V:$ A bacterial electron-bifurcating hydrogenase. J Biol Chem 2012, 287(37):31165-31171.

30. Li F, Hinderberger J, Seedorf H, Zhang J, Buckel W, Thauer RK: Coupled ferredoxin and crotonyl coenzyme a $(\mathrm{CoA})$ reduction with $\mathrm{NADH}$ catalyzed by the butyryl-CoA dehydrogenase/Etf complex from Clostridium kluyveri. J Bacterio/ 2008, 190(3):843-850.

31. Haldenwang WG: The sigma factors of Bacillus subtilis. Microbiol Rev 1995, 59(1):1-30.

32. Shao XJ, Raman B, Zhu MJ, Mielenz JR, Brown SD, Guss AM, Lynd LR: Mutant selection and phenotypic and genetic characterization of ethanol-tolerant strains of Clostridium thermocellum. Appl Microbio Biotechnol 2011, 92(3):641-652.

33. Miller EN, Jarboe LR, Turner PC, Pharkya P, Yomano LP, York SW, Nunn D, Shanmugam KT, Ingram LO: Furfural inhibits growth by limiting sulfur assimilation in ethanologenic Escherichia coli Strain LY180. Appl Environ Microbiol 2009, 75(19):6132-6141.

34. Paredes CJ, Alsaker KV, Papoutsakis ET: A comparative genomic view of clostridial sporulation and physiology. Nat Rev Microbiol 2005, 3(12):969-978.

35. Mearls EB, Izquierdo JA, Lynd LR: Formation and characterization of non-growth states in Clostridium thermocellum: spores and L-forms. BMC Microbiol 2012, 12:180

36. Fawcett $P$, Eichenberger $P$, Losick $R$, Youngman $P$ : The transcriptional profile of early to middle sporulation in Bacillus subtilis. Proc Natl Acad Sci U S A 2000, 97(14):8063-8068

37. Shi Z, Blaschek HP: Transcriptional analysis of Clostridium beijerinckii NCIMB 8052 and the hyper-butanol-producing mutant BA101 during the Shift from acidogenesis to solventogenesis. Appl Environ Microbiol 2008, 74(24):7709-7714.

38. Tomas CA, Alsaker KV, Bonarius HPJ, Hendriksen WT, Yang H, Beamish JA, Paredes CJ, Papoutsakis ET: DNA array-based transcriptional analysis of asporogenous, nonsolventogenic Clostridium acetobutylicum strains SKO1 and M5. J Bacteriol 2003, 185(15):4539-4547.
39. Hoch JA: Regulation of the phosphorelay and the initiation of sporulation in Bacillus subtilis. Annu Rev Microbiol 1993, 47:441-465.

40. Al-Hinai MA, Jones SW, Papoutsakis ET: sigmaK of Clostridium acetobutylicum is the first known sporulation-specific sigma factor with two developmentally separated roles, one early and one late in sporulation. J Bacteriol 2014, 196(2):287-299.

41. Fineran PC, Charpentier E: Memory of viral infections by CRISPR-Cas adaptive immune systems: acquisition of new information. Virology 2012, 434(2):202-209.

42. Raman B, Pan C, Hurst GB, Rodriguez M, McKeown CK, Lankford PK, Samatova NF, Mielenz JR: Impact of pretreated switchgrass and biomass carbohydrates on Clostridium thermocellum ATCC 27405 cellulosome composition: a quantitative proteomic analysis. PLoS One 2009, 4(4):e5271

43. Dror TW, Morag E, Rolider A, Bayer EA, Lamed R, Shoham Y: Regulation of the cellulosomal celS (cel48A) gene of Clostridium thermocellum is growth rate dependent. J Bacteriol 2003, 185(10):3042-3048.

44. Nicolaou SA, Gaida SM, Papoutsakis ET: A comparative view of metabolite and substrate stress and tolerance in microbial bioprocessing: from biofuels and chemicals, to biocatalysis and bioremediation. Metab Eng 2010, 12(4):307-331

45. Zhang $Y$, Han B, Chukwuemeka Ezeji T: Biotransformation of furfural and 5-hydroxymethylfurfural (HMF) by Clostridium acetobutylicium ATCC 824 during butanol fermentation. New Biotechnol 2011, 29(3):345-351.

46. Stern S, Dror T, Stolovicki E, Brenner N, Braun E: Genome-wide transcriptional plasticity underlies cellular adaptation to novel challenge. Mol Syst Biol 2007, 3:106.

47. Almeida JRM, Modig T, Petersson A, Hahn-Hagerdal B, Liden G, Gorwa-Grauslund MF: Increased tolerance and conversion of inhibitors in lignocellulosic hydrolysates by Saccharomyces cerevisiae. J Chem Technol Biotechnol 2007, 82(4):340-349.

48. Wang Q, Venkataramanan KP, Huang H, Papoutsakis ET, Wu CH: Transcription factors and genetic circuits orchestrating the complex, multilayered response of Clostridium acetobutylicum to butanol and butyrate stress. BMC Syst Biol 2013, 7:120.

49. Yu TT, Xu XP, Peng YF, Luo YM, Yang KQ: Cell wall proteome of Clostridium thermocellum and detection of glycoproteins. Microbiol Res 2012, 167(6):364-371.

50. Janssen H, Grimmler C, Ehrenreich A, Bahl H, Fischer RJ: A transcriptional study of acidogenic chemostat cells of Clostridium acetobutylicum-solvent stress caused by a transient n-butanol pulse. J Biotechnol 2012, 161(3):354-365.

51. Schwarz KM, Kuit W, Grimmler C, Ehrenreich A, Kengen SWM: A transcriptional study of acidogenic chemostat cells of Clostridium acetobutylicum - cellular behavior in adaptation to n-butanol. J Biotechnol 2012, 161(3):366-377.

52. Fujita $Y$, Matsuoka $H$, Hirooka K: Regulation of fatty acid metabolism in bacteria. Mol Microbiol 2007, 66(4):829-839.

53. Xu CG, Huang RR, Teng L, Wang DM, Hemme CL, Borovok I, He Q, Lamed R, Bayer EA, Zhou JZ, Xu J: Structure and regulation of the cellulose degradome in Clostridium cellulolyticum. Biotechnol Biofuels 2013, 6:15.

54. Bullard JH, Purdom E, Hansen KD, Dudoit S: Evaluation of statistical methods for normalization and differential expression in mRNA-Seq experiments. BMC Bioinformatics 2010, 11:94.

55. Wilson CM, Rodriguez M Jr, Johnson CM, Martin SL, Chu TM, Wolfinger RD, Hauser $\mathrm{L}$, Land ML, Klingeman DM, Syed MH, Ragauskas AJ, Tschaplinski TJ, Mielenz JR, Brown SD: Global transcriptome analysis of Clostridium thermocellum ATCC 27405 during growth on dilute acid pretreated Populus and switchgrass. Biotechnol Biofuels 2013, 6(1):179.

56. Tatusov RL, Galperin MY, Natale DA, Koonin EV: The COG database: a tool for genome-scale analysis of protein functions and evolution. Nucleic Acids Res 2000, 28(1):33-36

57. Morris JA, Gardner MJ: Calculating confidence intervals for relative risks (odd ratios) and standardised ratios and rates. Br Med J 1988, 296(6632):1313-1316.

doi:10.1186/s12866-014-0215-5

Cite this article as: Linville et al:: Transcriptomic analysis of Clostridium thermocellum Populus hydrolysate-tolerant mutant strain shows increased cellular efficiency in response to Populus hydrolysate compared to the wild type strain. BMC Microbiology 2014 14:215. 